\title{
1 EVIDENCE FOR ALTERNATE STABLE STATES IN COLLAPSING 2 ECOLOGICAL NETWORKS
}

3 (Running Title: Multiple stable states in networks)

4 Suresh $\mathrm{Babu}^{1}$ and Gitanjali Yadav ${ }^{2}$

5 Email Addresses: (SB)suresh@aud.ac.in; (GY) gy@nipgr.ac.in;

\section{Affiliations:}

$7{ }^{1}$ School of Human Ecology, Ambedkar University of Delhi, Kashmere Gate, Delhi,

$8 \quad$ India 110006.

$9 \quad{ }^{2}$ Genomics \& Systems Biology Laboratory, National Institute of Plant Genome

10 Research (NIPGR), Aruna Asaf Ali Marg, New Delhi, India 110067.

12 KeyWords: Ecological networks, Network Attributes, Extinction cascade, Network

13 Diameter, Alternate Stable States, Resilience

15 To Whom Correspondence should be addressed:

16 Dr. Suresh Babu

17 School of Human Ecology

18 Ambedkar University of Delhi,

19 Kashmere Gate,

20 Delhi,

21 India 110006.

22 Email: suresh@aud.ac.in 


\section{ABSTRACT}

2 Background: There has been considerable interest and progress in our perception of

3 organized complexity in recent years. Recurrent debates on the dynamics and stability

4 of complex systems have enriched our understanding of these systems, but

5 generalities in the relationship between structure and dynamics are hard to come by.

6 Although traditionally an arena for theoreticians, much of this research has been

7 invigorated by demonstration of the existence of alternate stable equilibria in real

8 world ecosystems such as lakes, coral reefs, forests and grasslands.

9 Results: Linking up systems thinking with recent advances in our understanding of

10 ecological networks opens up exciting possibilities. In an attempt to obtain general

11 patterns of behaviour of complex systems, we have analyzed the response of eighty-

12 six real world ecological networks to targeted extinctions, and the findings suggest

13 that most networks are robust to loss of specialists until specific thresholds are

14 reached in terms of geodesics. If the extinctions persist, a state change or 'flip' occurs

15 and the structural properties are altered drastically, although the network does not

16 collapse. Further, we find that as opposed to simpler networks, larger networks have

17 several such alternate states that ensure their long-term persistence and that indeed

18 complexity does endow resilience to such networks.

19 Conclusions: This is the first report of critical transitions in ecological networks and

20 the implications of these findings for complex systems characterized by networks are

21 likely to be profound with immediate significance in conservation biology, invasion

22 biology and restoration ecology. 


\section{BACKGROUND:}

2 Interest in 'robust, yet fragile' nature of complex systems transcends disciplinary

3 domains of biology, engineering, sociology, economics, and ecology[1,2]. There is

4 much to be gained by investigating the behavior of unique complex dynamical

5 systems like ecosystems that are robust by virtue of their continued existence in

6 evolutionary time[2]. The structural attributes shared by these systems could provide

7 clues about their stability and robustness. Systems such as scale free ecological webs

8 display an unexpected degree of tolerance or structural robustness to loss of

9 specialists[3,4]. Studies on mutualistic networks have highlighted that modularity-

10 one of the emergent properties of networks, endows robustness[5]. Be it fire prone

11 savanna ecosystems, spread of infectious diseases or financial networks like the

12 Fedwire, compartmentalization has been shown to render the much needed robustness

13 to these systems[2]. The dynamics of large complex networks formed by interacting

14 species impact the way biodiversity influences ecosystem functioning[6].

15 Understanding the behaviour of ecological networks is also central to understanding

16 the response of biodiversity and ecosystems to perturbations.

17 Although there is adequate evidence to imply that structural and topological attributes

18 of networks influence dynamics and function[7,8,9], the attributes of nodes and

19 overall topological properties of networks that endow stability against perturbations

20 are not sufficiently understood. The 'targeted extinction' approach for exploring the

21 effects of node loss and associated co-extinctions has been well established over the

22 last decade[3,10,11]. This approach involves simulations of random or ordered

23 primary extinctions based on a given node property such as the number of links or

24 'degree'. The response of the network in terms of resulting secondary extinctions or

25 other network properties can be used to infer the significance of the node attribute 
1 being studied[4,12]. Extensive work on the robustness of ecological networks and

2 attributes that enable species coexistence[5] and diversity[9] have revealed that these

3 networks are highly robust to loss of specialists but are unable to withstand the

4 targeted removal of generalists. This study was undertaken with the aim to understand

5 how species persist in a collapsing mutualistic network following targeted species

6 removals and associated coextinctions. The initial analysis was carried out using

7 primary data on frugivory collected from Great Nicobar Island, India (GNIC) and the

8 response and behavior of the network was explored in terms of established network

9 attributes including species richness, secondary extinctions, nestedness, fragmentation

10 and diameter. The analysis led to the discovery of alternate stable states that help

11 sustain the integrity of the collapsing network. These alternate states are identifiable

12 in terms of two attributes describing internal communication within the network,

13 namely, its diameter (LDia) and number of diameters (NDia). A comparative analysis

14 using extinction cascades across eighty-five ecological networks of varying nature

15 was used to validate the observations made with GNIC, which revealed that these

16 alternate states and flips were indeed pervasive across all the networks.

\section{RESULTS}

The GNIC is a single large connected network having 812 interactions between 38 frugivores and 181 tree species (binary interaction matrix in Supplementary Data

21 A1), making it one of the largest frugivory networks reported to date. Figure 1a represents an edge weighted, force directed layout of GNIC depicting highly

23 asymmetric interactions, a characteristic path length of three, and a diameter of six. A

24 radial layout of GNIC showed that species interact with nested subsets of partners. As 25 expected of ecological networks, the nestedness of GNIC is high (NodF value 21.02) 
1 and its degree distribution showed best fit to a truncated power law distribution

2 (Figure $1 \mathrm{~b}$ and $\mathrm{c}$ ). In comparison with other frugivory webs reported to date, GNIC

3 has higher links per species (L/S), greater density, asymmetry and specialization, and

4 a comparatively lower Connectance $\left(\mathrm{L} / \mathrm{S}^{2}\right)$.

Degree based co-extinction simulations were carried out for GNIC, and the

7 response was observed using different network level indices. As species removal is

8 simulated from the most specialized (least-linked) to most-generalized (most-linked),

9 along a specialist-generalist continuum, species richness decreases linearly and

10 secondary extinctions do not occur until $61 \%$ primary extinctions. This can be seen in

11 Figures 2a-b, which also depict the opposing 'generalists-first' extinction sequence,

12 resulting in a sharp decrease in species richness accompanied by drastic secondary

13 extinctions. This contrasting response was also observed in terms of the overall

14 change in nestedness after perturbation; as specialists are removed, nestedness of the

15 resulting networks tends to increase, while the removal of generalists triggers a rapid

16 loss of nestedness in the corresponding reduced webs (Figure 2c). Notably, GNIC was

17 able to sustain its unfragmented nature throughout the specialist-first extinction

18 sequence, disintegrating only after removal of over $90 \%$ nodes; whereas, the reverse

19 sequence (generalist first extinction) resulted in catastrophic network fragmentation

20 into many disconnected sub-webs and complete collapse within the first $23 \%$ primary

21 species removals (Figure 2d).

To explain the observed robustness of GNIC to loss of specialists, we carried

23 out a detailed examination of the two contrasting breakdown scenarios, in terms of 
1 additional bipartite network attributes such as degree-distribution exponent gamma,

2 density, asymmetry, connectance, generality, specialisation, C-score, V-ratio and

3 various aspects of geodesics, including number, length and unique sets of shortest

4 paths. Although for most of these properties, patterns were not discernable, two

5 mutually independent attributes describing internal network communication (LDia

6 and NDia) showed striking structural breaks (Figure 2e and f). Most prominently,

7 these two attributes show a coordinated response when specialists are removed,

8 revealing a characteristic pattern that is absent in case of generalists-first extinctions.

9 As can be seen, in the generalists-first extinction sequence, LDia briefly increases

10 followed by a steep decrease and rapid collapse, a trend that corresponds with NDia

11 curves; loss of generalists leads to a brief but drastic increase in NDia (to over 36900

12 at just under 5\% primary extinctions), after which it steeply drops (to 455 by $10 \%$

13 deletions). This low number of diameters (NDia) corresponds to a failure of internal

14 communication and subsequently the network undergoes fragmentation. A

15 comparison of the plots (Figure $2 \mathrm{~d}$ and e) shows that the first instance of

16 fragmentation in the collapsing network occurs at about $10 \%$ deletions, coinciding

17 exactly with the lowest value of NDia. Further node deletions rapidly result in more

18 fragments and the network collapses by $23 \%$ removals. In contrast, when specialists

19 are removed first, LDia remains constant and NDia decreases steadily. By about 8\%

20 deletions, NDia reaches its lowest value of 436. However, the plot in Figure 2d shows

21 that despite minimal internal communication, the single unit connected character of

22 the collapsing network is preserved. Interestingly, the lowest value of NDia

23 corresponds to a single unit reduction in the LDia, which in turn, results in a steep

24 recovery of NDia values (from 436 to 25966). Subsequently this pattern repeats itself,

25 i.e, NDia decreases at pace with loss of specialists till about $80 \%$ extinctions. At its 
1 lowest value, it drastically rises again - corresponding to a further unit reduction in

2 LDia. Evidently, the coordinated response between NDia and LDia and the associated

3 renewal of internal communication, makes it possible for the reduced network to

4 make a stable transition to a new state and remain unfragmented, all through the

5 specialist-first extinction sequence. Such a compensatory 'flip' response between two

6 network attributes, specific to the specialist-first scenario, and absent in the generalist-

7 first scenario, has not been reported before. Supplementary Data Sheet A3 provides

8 details of this analysis, and the number of fragments, co-extinctions, LDia and NDia

9 measured after every consecutive species deletion, both for the specialist-first and the 10 generalist-first extinction cascades.

\section{Comparative Analysis of Ecological Networks}

A comparative analysis of 85 additional ecological networks showed these

13 patterns in LDia, NDia and fragmentations to be consistent and pervasive across all networks during the specialists-first breakdown scenario, and not limited to mutualistic webs only. As with GNIC the coordinated variation between NDia and LDia values endowed robustness to the perturbed networks and they persisted as

17 single connected units during the attacks. In several cases, the different states were 18 more pronounced than observed for GNIC. At least two and upto six flips were 19 observed across the networks. In networks with low interaction density $(<1.35)$, the 20 transition between states was not very clear. In cases where the initial network was 21 disconnected, the specialist-first extinction sequence began with the removal of the 22 smaller unit/s, and eventual persistence of the single largest unit. Figures $3 a$ and $b$ 23 depict the synchronised behaviour observed in two of the 25 frugivory webs studied 24 (codes SILV and JOR1, see Supplementary Data for details). Figure 3c shows the 
1 flips observed in MEMO, a pollination network with 299 interactions among 104

2 species. Figures 3d and e depict similar plots for an anemone-fish network (ANEM),

3 and an ant-plant network (BLUT) respectively. Figures $3 \mathrm{f}-\mathrm{h}$ depict the LDia-NDia

4 plots for a host-parasite, plant-herbivore and a predator-prey network (LAKE, JEOM

5 and MART) respectively.

For all networks, 'resistance to flip' was estimated in terms of percentage of

7 primary species extinctions after which the first flip was observed. Therefore

8 'resistance to flip' is higher if a large number of species deletions are required before

9 the flip is observed, and lower if fewer node deletions cause the state change. Figure

10 4a shows the relationship between the 'resistance to flip' and initial LDia across the

1186 ecological networks. Compact networks with small diameters require over $70 \%$

12 primary extinctions for a flip in their state (Figure 4a). Networks with initial diameters

13 of 6 or more require a much smaller proportion of primary extinctions to switch to

14 lower diameters. A linear regression of 'resistance to flip' on initial LDia indicates a

15 significant negative relationship (Adjusted $R^{2}=0.3598, \beta=-7.455, \mathrm{df}=84, \mathrm{p}$-value:

16 6.288e-10). However a Loess plot of the same indicates a curvilinear rather than a

17 linear relationship between the variables, when examined at different spans (Figure

18 4b). The data was found to meet the assumptions of homoscedasticity and normality

19 of errors (Supplementary Figure S1). It was also observed that a positive, nearly linear

20 relationship exists between the likely number of flips and the initial diameter of the

21 network (Linear model; Adjusted $\mathrm{R}^{2}=0.5981, \beta=0.559$, $\mathrm{df}=84$, $\mathrm{p}$-value: $<2.2 \mathrm{e}-16$;

22 Supplementary Figure S2). Results of the entire analysis of 86 ecological networks 23 are presented in Supplementary Data A. 
1 Ecological networks are well known to be robust to removal of specialists, but

2 reveal an intrinsic structural fragility in response to targeted removal of generalists,

3 eventually resulting in fragmentation into many small sub-webs. This behavior is not

4 shared with random networks, which are equally fragile to random or selective node

5 removals[13]. We began this work with GNIC frugivory data, a new network showing

6 characteristic features similar to known ecological webs, to investigate the contrasting

7 ability of mutualistic networks to withstand attacks on specialists as against

8 generalists, the former known to be a more realistic extinction threat. As expected,

9 distinct responses were observed. Generalist first extinction cascade of GNIC caused

10 the species richness to plummet due to steep rise in coextinctions, whereas the

11 specialist first cascade shows a linear decrease in species richness as it does not

12 involve the loss of associated species. Nestedness, one of the most significant and

13 widely observed non-random pattern in networks of ecological interactions, is known

14 to greatly affect the robustness of mutualitsic networks[14]. The variation in the

15 nested structure of the reduced webs in the two contrasting scenarios revealed that as

16 specialists are removed, nestedness of the reduced network increases, while the

17 removal of generalists resulted in a steep decrease in nestedness, supporting the

18 notion that nestedness provides alternate routes for system responses after

19 perturbations such as species extinctions or link removals. Nestedness being a 20 measure of robustness[9,14] this indicates that extinctions of specialists improve the

21 robustness of the reduced networks. Of the several structural attributes analysed to

22 understand this ability, the most striking patterns emerged from the behaviour of the

23 geodesics as the network was subjected to systematic extinctions. 
1 The coextinction cascades simulated with GNIC showed that with specialists

2 being removed first, the graph does not fragment unless substantial primary

3 extinctions have occurred. Our initial findings suggested that in the specialist-first

4 scenario, network attributes 're-wire' to make the reduced network more compact,

5 thereby maintaining optimal communication between the remaining nodes, keeping

6 the network unfragmented. These topological re-adjustments are characteristic and

7 were identified in terms the network diameter, which represents the longest geodesic

8 of the network. Although the diameter has been relatively less studied in mutualistic

9 webs, it is a well established measure of topological robustness of several complex

10 communication systems, ranging from cells to social, civilian networks and the

11 Internet[10,15]. For a given network, a low diameter is considered advantageous as it

12 can contribute to greater interconnectedness, shorter communication paths and lower

13 load on links, or edges. We examined two aspects of the diameter: (a) its length or

14 'LDia', and (b) the number of diameters or 'NDia', and discovered a striking

15 synchrony between LDia and NDia in the specialist-first extinction scenario,

16 presumably an internal compensation that endows the perturbed network with the

17 ability to avoid fragmentation. Every instance of a very low NDia value was found to

18 coincide exactly with a corresponding reduction in the LDia value, leading to a

19 reversal of the decreasing NDia trend. A sufficient number of NDia in the collapsing

20 network presumably enable it to maintain communication between remaining nodes,

21 which remain connected despite the sustained perturbations. This coupling was not

22 observed in the generalist-first extinction sequence, where the perturbed network,

23 unable to recover after an excessive decrease in its NDia, undergoes multiple

24 fragmentations. 
We have attempted to explain the contrasting responses in the two breakdown

2 scenarios using a schematic in Supplementary Figure S3. As shown in this Figure, the

3 generalist-first extinction sequence begins with a loss of crucial hubs through which

4 the original diameter-paths were running, and this deletion leads to the selection of

5 longer routes, causing the LDia to increase at first. Further loss of hubs then causes

6 the network undergo fragmentation into clusters of nodes, whose sizes (and

7 corresponding LDia) decrease rapidly with continued node removal, until total

8 collapse. In contrast, when the specialists are removed first, the nodes break away one

9 by one from the periphery, rather than as clusters, so that a large number of alternate

10 paths of length LDia remain available. As a result, the NDia slowly decreases while

11 the LDia remains constant. As NDia reaches its lowest value, the LDia flips and

12 becomes smaller. This reduction in LDia causes an abrupt rise in NDia is periodic and

13 enables the network to avoid fragmentation when the NDia reaches extremely low

14 values. We ascribe this rise in NDia to the combined number of (a) pre-existing paths

15 at the new (reduced) LDia, and (b) the node loss driven shorter paths created from the

16 previous (larger) LDia. This LDia - NDia synchrony repeats itself all along the

17 breakdown scenario although on a progressively smaller scale. Consequently, the

18 network displays high topological stability throughout the extinction cascade by

19 remaining un-fragmented.

The iterating pattern of gradual decrease in NDia till a threshold of extinctions

21 is reached, followed by a sudden transition to a new high value at a lower LDia,

22 resembles the behaviour of ecosystems that can exist in multiple states characterized

23 by unique sets of conditions[16,17,18]. The theory of alternate stable states suggests

24 that the discrete states are separated by thresholds and the system remains in one state

25 unless perturbation is large enough to tip it over to the next state[16,19]. It has been 
1 suggested that gradual changes are more like the rule and critical transitions are an

2 exception, which demand special attention[20]. In case of GNIC, the network retains

3 its integrity by flipping between alternate levels of communication and complexity

4 expressed in terms of LDia. However for the reduced network, the increased NDia

5 now endows the system with high resilience, as the threshold required for the next flip

6 or shift in LDia requires over $60 \%$ primary extinctions. The state with the widest

7 stability basin, characterized by the maximum range of NDia at a given LDia,

8 provides much of the robustness of the network. Figure 5 depicts this in a schematic

9 representation. Hysteresis or path dependency, characteristic of alternate equilibria,

10 becomes evident once a flip in LDia has occurred. If the lost node were to be returned

11 to the network at this stage, it may not bring the system back to the previous state.

12 Rather, it would lead to an increase in the NDia within the current state, i.e at the new

13 value of LDia. This is likely because of the increasingly nested pattern of the reduced

14 networks (Figure 2c) a new species is likely to preferentially attach to the 'hub'

15 nodes or generalists[6,21,22]. Attachment to a hub node does not lead to an increase

16 in LDia; it can only result in additional alternate paths or NDia. As a result, the

17 system will not return to its previous state just by a simple reversal of extinction, or

18 re-introduction of lost species. This observation may have wide implications in the

19 area of restoration ecology and invasion biology, as we discuss later.

The following generalizations emerge from our observations on GNIC: (a) total

21 number of alternate paths or diameters (NDia) decrease with loss of specialists, (b) for

22 a given network, reduction in the diameter (LDia) increases the NDia, (c) the LDia

23 reduction occurs only at, or beyond, a critical loss of specialists, (d) the network

24 precludes fragmentation, with the loss of specialists across the entire cascade and (e)

25 for a given network, there may be several alternate stable states that can spring 
1 surprises against slow moving perturbations which can be masked by internal

2 adjustments of the network.

The generality of these observed patterns in LDia-NDia was established by a

4 comparative analysis across 85 additional ecological networks including mutualisms

5 as well as antagonistic. webs. Our results show that the collapsing network sustains its

6 connected or un-fragmented nature during the loss of specialists by internal structural

7 readjustments in terms of LDia and NDia, which is not evident during the loss of

8 generalists, thereby leading to immediate collapse. We also find that initial network

9 size corresponds to the number of flips observed. Larger networks are likely to have

10 more number of alternative stable states to cope with uncertainties in evolutionary

11 time. For example, a small network like the anemone-fish network has only 36

12 species and an unperturbed LDia of 4, resulting in only one alternate stable state

13 which may restrict its ability to withstand perturbations (Figure 3d). Larger networks

14 like the Brazilian Amazon (code SILV) and GNIC have several possible alternate

15 stable states and are more likely to persist under long periods of adversity. Smaller

16 perturbations tend to flip larger, more complex networks to alternate states (Figure 4a

17 and b) and since they have several such possible states, the network architecture

18 endows resilience to such networks. The smaller, less complex networks do not show

19 any state changes under small perturbations indicating resistance. However, since

20 smaller networks also have very few possible alternate states, they are low on

21 resilience. The width of stability basins and the number of possible stable states that

22 accompanies the loss of specialists progressively shrinks, as the network size reduces,

23 thus affecting its overall resilience. Therefore there may be an evolutionary advantage

24 in making ever larger webs of interactions that facilitate long-term persistence of 
1 species rich communities, a finding that complements a recent study[9] as to how

2 mutualistic communities can enhance co-existence of species.

\section{Implications of alternate states in ecological networks}

Co-extinctions are now recognized as a major driver of global biodiversity loss,

6 along with habitat destruction, species invasion and overkill [23,24]. Since more than

7 half of all known species and a large proportion of unnamed ones are involved in host

8 specific relationships in atleast some stage of their life, specialists face a greater risk

9 due to secondary extinctions[25,26]. There is added relevance of re-examining the

10 threats of extinction knowing that interacting species may exist in alternate states.

11 Besides broad implications on our understanding of bipartite networks in general, our

12 findings have significance in conservation biology, invasion biology, and restoration

13 ecology. Based on the present positioning of an interaction network along an

14 extinction cascade, it may be possible to predict the proximity of the system to a

15 catastrophic change and model real time stability indicators of networks. In addition

16 to the dynamics associated with 'critical slowing down[27], this may be an alternate

17 approach to predict the likelihood and proximity of a system to regime flips.

18 Conservation programmes could benefit from directly identifying the most threatened

19 systems, requiring immediate attention or prioritization.

High nestedness and NDia of the reduced networks can make the system

21 receptive to invasive species because of the benefits associated with joining a well-

22 connected network. Invasive species have been shown to be able to take advantage of

23 existing mutualistic networks in invaded habitats[28] and the state of the native

24 network may explain its invasibility. A native seed dispersal network in which most

25 of the specialists have been removed is more vulnerable to invasion as the invader 
1 would be able to associate with a generalist without much competition from other

2 specialists. This provides the invader with enhanced connectivity, which has a vital

3 role in its persistence and spread. Coupled with experimental evidences[22] our

4 findings pave the way for developing a network approach to invasion biology.

Hysteresis in collapsing networks as implied in the theory of alternate stable

6 states has for instance profound implications in restoration ecology. It may be

7 possible to explain why certain restoration programmes do not follow expected

8 trajectories and one may aspire to find system specific predictors of thresholds of

9 recovery. Path dependency of collapsing networks informs us about the near

10 impossibility of reconstructing highly degraded ecosystems.

11 Another functional consequence of such a pervasive phenomenon would be on

12 the ability of networks to transmit or 'percolate' perturbations across the network.

13 Coupled oscillations are likely to travel far and wide across the network much more

14 effectively as LDia decreases. In real ecosystems, this could have major implications.

15 A drastic reduction in the abundance of a particular species owing to hunting or

16 disease would impact the network much faster in unpredictable ways. This would

17 again imply an increased uncertainty over the behaviour of such networks. The flips

18 in diameter following loss of specialists could well upset the functional advantages of

19 scale free networks[10,13]. The outward robustness of scale free networks to loss of

20 specialists could mask the enhanced ability of the network to transmit perturbations.

\section{CONCLUSIONS}

Our results provide empirical evidence for the direct link that exists between

23 topological heterogeneity and system dynamics. We show by means of detailed 
1 analysis of eighty-six ecological networks of varying nature that the networks can

2 exist in alternate diameters and levels of communication. The outwards stability and

3 unfragmented nature of these networks against perturbations often mask the internal

4 re-wiring that progressively reduces their resilience resulting in sudden flips or

5 transitions to lower levels of communication. This study shows that the continuous

6 loss of specialists leads to significant loss of resilience for the networks, which is

7 irreversible - something impossible to demonstrate experimentally. On one hand these

8 findings hint at an evolutionary advantage in building ever-larger interaction networks

9 (moving to higher levels of robustness), and on the other hand also highlights the

10 inability of heavily damaged networks to respond to restoration in tangible amounts of

11 time. The increased likelihood of an invasive species attaching to generalists in an

12 impoverished native network partly explains its success in invaded ecosystems. The

13 robustness of scale free networks could disguise enhanced percolation of disturbances

14 across the network. This study establishes a prevailing pattern across known complex

15 ecological networks and open ups possibilities for empirically driven dynamical

16 modelling of these networks. We expect our findings to be the starting point for an

17 array of investigations into the importance of alternate states in ecological networks in 18 particular and other kinds of networks in general.

\section{MATERIALS \& METHODS}

Primary data in the form of direct observations of foraging by vertebrates on

23 fruits was collected from the tropical rainforests of Great Nicobar Island (spread from

$246^{\circ} 45^{\prime}$ to $7^{\circ} 15^{\prime} \mathrm{N}$ and $93^{\circ} 38^{\prime}$ to $93^{\circ} 55^{\prime} \mathrm{E}$, spanning a total area of about $1045 \mathrm{~km}^{2}$ ), the 
1 southernmost Island in the Andaman \& Nicobar archipelago, India, spanning a period

2 of seven years with field work being conducted on fifty nine transects, each $500 \mathrm{~m}$

3 long, in various regions of the island from December 1999 to November 2006. This

4 study was undertaken as part of a larger initiative by the Ministry of Environment and

5 Forests, Government of India, under the Man and Biosphere (MAB) Programme on

6 Great Nicobar Biosphere Reserve, India. Direct observations of instances of foraging

7 by vertebrates on fruits were recorded as an interaction matrix consisting of 181 plant

8 species and 38 frugivores ( 33 birds and 5 mammals). Plant and frugivore species were

9 identified and the interaction data obtained was compiled for the entire island. Data is 10 presented as a binary interaction matrix (Supplementary Data A1). Preliminary 11 analysis and visualization of network architecture was done using Cytoscape [29] 12 version 2.6.2.

13 Co-extinction Analysis

14 We simulated primary species loss by carrying out cascades of directed 15 species removals or extinctions, based upon degree (the number of links), and 16 compared the stability of the resulting reduced networks to random extinction 17 cascades, following Memmott et al[3]. Upon removal of a species, those species that 18 are left without any interaction are assumed to undergo co-extinction. The network 19 remaining after each subsequent removal is assessed for robustness and stability. For 20 each network, extinction sequences included both specialist-first (i.e least-linked to 21 most-linked species) and generalist-first (most-linked to least linked species) 22 cascades. Random removals were analysed after averaging from 300 replicates. The network remaining after every primary extinction and subsequent co24 extinction was analysed for its stability and robustness by measuring various network 25 attributes commonly used to summarise and describe different patterns in ecological 
1 webs, such as degree, species richness, secondary extinction, fragmentation, lost

2 interactions, degree-distribution-gamma values, axes, length and number of diameters

3 etc. These indices were calculated using in-house fortran scripts and R CRAN

4 packages IGRAPH[30] version 0.5, SNA[31] version 1.5, and BIPARTITE[32]

5 version 0.91 . Detailed description of each of the indices can be found within the

6 respective package manuals. We examined the exponential, power law and truncated

7 power law models to cumulative distributions for each network. Nestedness was

8 calculated using the recently proposed nestedness metric NodF[33] using the

9 ANINHADO program[34]. To assess the significance of nestedness values, the

10 observed NodF was compared with benchmarks provided by three different null

11 models. For each network, a population of $n=300$ random networks was generated

12 for each null model. As a statistic indicating significance, we estimated the

13 probability, $\mathrm{p}$, that a randomization was equally or more nested than the real matrix.

14 Only the significant NodF values were used for further analysis. Comparison of

15 nestedness across reduced networks was done without normalizing these values for

16 variation in species richness or number of interactions, since each reduced network is

17 essentially a subset of the original unperturbed network. The shortest paths (also

18 called geodesics) were calculated by using breadth-first search in the graph. The

19 diameter (LDia) of a graph is defined as the length of the longest geodesic. The

20 number of diameters (NDia) was calculated as the sum of all diameters between every

21 pair of nodes separated by a distance equivalent to the diameter (LDia). A unix

22 program was designed to automate the entire analysis. This code takes a given binary

23 network as input, simulates different co-extinction sequences and evaluates the sub-

24 network remaining after every subsequent species removal, for its stability and

25 robustness, and then extracts the attributes required for detection of regime flips or 
1 alternate stable states. For each reduced network, it creates a list of extinct and co-

2 extinct species and calculates seven network level indices, namely species richness,

3 secondary extinction, lost and remaining interactions, number of fragments, LDia and

4 NDia and compares these indices across and between the different extinction

5 sequences, and finally plots the results into vector format files. The source code is

6 currently being developed as an open source web server facility.

In addition to GNIC, data records were obtained from a set of 85 ecological networks using previously published reports as well as the Interaction Web Database

9 repository at the National Centre for Ecological Analysis and Synthesis (NCEAS)

10 website (http://www.nceas.ucsb.edu/interactionweb). These 85 webs include one

11 Anemone Fish network, four plant-herbivore, four ant-plant, seven host-parasite, one

12 Predator-prey, 25 Seed dispersal or Frugivory networks and 43 Pollination networks.

13 Each network was analysed using the code described above and subsequently

14 examined for the occurrence of regime flips, as they appeared on plots of NDia and

15 LDia with primary extinctions. Statistical analyses on the results across networks

16 were carried out in R (V 2.11.0). Supplementary Table S1 contains a brief description

17 of these networks and details with references are in Supplementary Data Sheet A2.

\section{LIST OF ABBREVIATIONS}

\section{ACKNOWLEDGEMENTS}

21 S.B acknowledges the Man \& Biosphere (MAB) Programme of the Ministry of

22 Environment and Forests, Govt of India, for support. G.Y thanks Director, NIPGR for 23 support. 


\section{FINANICAL DISCLOSURE}

2 S.B acknowledges the Man \& Biosphere (MAB) Programme of the Ministry of

3 Environment and Forests, Govt of India, for financial and logistic support. G.Y thanks

4 the Dept of Biotechnology (DBT), Govt. of India for funding. S.B acknowledges the

5 research fellowship of Council for Scientific \& Industrial Research (CSIR) in the $6 \quad$ initial years of this project.

\section{AUTHOR CONTRIBUTIONS}

8 S.B designed the study and compiled the primary data used in the analysis. G.Y

9 performed the computational simulations and wrote the co-extinction code used in 10 this study. S.B and G.Y jointly analyzed the results and wrote the first version of the 11 manuscript, both authors contributed to the final draft.

\section{REFERENCES}

1. Carlson JM, Doyle J (2002) Complexity and robustness. Proc Natl Acad Sci U S A 99 Suppl 1: 2538-2545.

2. May RM, Levin SA, Sugihara G (2008) Ecology for bankers. Nature 451: 893-895.

17 3. Memmott J, Waser NM, Price MV (2004) Tolerance of pollinator networks to species extinctions. Proc R Soc Lond B 271: 2605-2611.

19 4. Srinivasan UT, Dunne JA, Harte J, Martinez ND (2007) Response of complex food webs to realistic extinction sequences. Ecology 88: 671-682.

21 5. Bascompte J, Jordano P, Olesen JM (2006) Asymmetric coevolutionary networks facilitate biodiversity maintenance. Science 312: 431-433.

23 6. Pascual M, Dunne JA (2006) Ecological Networks: Linking Structure to Dynamics in Food Webs. 
1 7. Montoya JM, Pimm SL, Sole RV (2006) Ecological networks and their fragility. Nature 442: 259-

2

3 8. Sugihara G, Ye H (2009) Complex systems: Cooperative network dynamics. Nature 458: 979-980. 264.

9. Bastolla U, Fortuna MA, Pascual-Garcia A, Ferrera A, Luque B, et al. (2009) The architecture of mutualistic networks minimizes competition and increases biodiversity. Nature 458: 10181020.

10. Albert R, Jeong H, Barabasi AL (2000) Error and attack tolerance of complex networks. Nature 406: 378-382.

11. Sole RV, Montoya JM (2001) Complexity and fragility in ecological networks. Proc Biol Sci 268: 2039-2045.

12. Dunne JA, Williams RJ (2009) Cascading extinctions and community collapse in model food webs. Philos Trans R Soc Lond B Biol Sci 364: 1711-1723.

13. Strogatz SH (2001) Exploring complex networks. Nature 410: 268-276.

14. Jordano PJ, Bascompte J, Olesen JM, Waser NM (2006) The ecological consequences of complex topology and nested structure in pollination webs. In: Waser NM, Ollerton J, editors. PlantPollinator Interactions: From Specialization to Generalization. Chicago, IL: University Of Chicago Press. pp. 173-199.

15. Dekker AH, Colbert BD. Network robustness and graph topology; 2004 January 01, 2004; Dunedin, New Zealand pp. 359 - 368.

16. May RM (1977) Thresholds and breakpoints in ecosystems with a multiplicity of stable states. Nature 269: 471-477.

17. Scheffer M (2001) Catastrophic shifts in ecosystems. Nature 413: 591-596.

18. Sutherland JP (1974) Multiple Stable Points in Natural Communities. The American Naturalist 108 : 859-873.

19. Scheffer M, Carpenter SR (2003) Catastrophic regime shifts in ecosystems: linking theory to observation. Trends Ecol Evol 18: 648-656.

20. Scheffer M (2009) Critical Transitions in Nature and Society. Princeton University Press.

21. Vazquez DP, Aizen MA (2004) Asymmetric specialization: a pervasive feature of plant-pollinator interactions. Ecology 85: 1251-1257. 
22. Memmott J, Waser NM (2002) Integration of alien plants into a native flower-pollinator visitation web. Proc Biol Sci 269: 2395-2399.

23. Diamond JM (1989) The present, past and future of human-caused extinctions. Philos Trans R Soc Lond B Biol Sci 325: 469-476; discussion 476-467.

24. Poulin R, Morand S (2000) The diversity of parasites. Q Rev Biol 75: 277-293.

25. Koh LP, Dunn RR, Sodhi NS, Colwell RK, Proctor HC, et al. (2004) Species coextinctions and the biodiversity crisis. Science 305: 1632-1634.

26. Dunn RR, Harris NC, Colwell RK, Koh LP, Sodhi NS (2009) The sixth mass coextinction: are most endangered species parasites and mutualists? Proc Biol Sci 276: 3037-3045.

27. Scheffer M, Bascompte J, Brock WA, Brovkin V, Carpenter SR, et al. (2009) Early-warning signals for critical transitions. Nature 461: 53-59.

28. Buckley YM, Anderson S, Catterall CP, Corlett RT, Engel T, et al. (2006) Management of plant invasions mediated by frugivore interactions. Journal of Applied Ecology 43: 848-857.

29. Shannon P, Markiel A, Ozier O, Baliga NS, Wang JT, et al. (2003) Cytoscape: a software environment for integrated models of biomolecular interaction networks. Genome Res 13: 2498-2504.

30. Csardi G, Nepusz T (2006) The igraph software package for complex network research. Complex Systems InterJournal 1695.

31. Butts CT (2008) Social Network Analysis with sna. Journal of Statistical Software 24.

32. Dormann CF, Frund J, Bluthgen N, Gruber B (2009) Indices, graphs and null models: analyzing bipartite ecological networks. The Open Ecology Journal 2: 7-24.

33. Almeida-Neto M, Guimarães P, Jr PRG, Loyola RD, Ulrich W (2008) A consistent metric for

34. Guimarães JPR, Guimarães P (2006) Improving the analyses of nestedness for large sets of matrices. Environmental Modelling \& Software 21: 1512-1513. nestedness analysis in ecological systems: reconciling concept and measurement. Oikos 117: 1227-1239.

\section{列}




\section{1. Supplementary Data A (EXCEL FORMAT FILE)}

2 This file contains Supplementary Data with sheets (A1) The Binary interaction data for GNIC

3 frugivory network reported in this paper (A2) listing of all 86 datasets and their references (A3)

4 detailed results of the co-extinction analysis on GNIC frugivory network, (A4) Summary results of the

5 entire analysis on 86 networks.

\section{2. Supplementary Data B (PDF FORMAT FILE)}

7 This file contains Supplementary Figures S1, S2, S3, Supplementary Table S1.

\section{AUTHOR CONTRIBUTIONS}

9 S.B designed the study and compiled the primary data used in the analysis. G.Y performed the 10 computational simulations and wrote the co-extinction code used in this study. S.B and G.Y jointly 11 analyzed the results and wrote the first version of the manuscript, and all authors contributed to the 12 final draft.

\section{COMPETING INTERESTS}

14 The authors declare no competing interests. Correspondence and requests for materials should be 15 addressed to S.B (suresh@aud.ac.in) 


\section{FIGURE LEGENDS}

2 Figure 1

3 Illustration of the GNIC frugivory network from Great Nicobar Island, India: (a)

4 Visual layout - circles represent species or nodes (green for plants; yellow for

5 animals) and lines or edges between nodes represent the interactions between species.

6 GNIC is a bipartite network with a diameter or LDia of 6. Four diameters are

7 highlighted in this figure as thick paths of length 6 , in blue, orange, red and pink. The 8 unperturbed network contains 10065 such diameters or NDia, i.e independent shortest

9 paths of length 6 , between a given pair of nodes, (b) The nested pattern of GNIC 10 matrix of interactions, and (c) Degree distribution of GNIC nodes, showing the best 11 fit to truncated power law curve.

12 Figure 2

13 GNIC response to targeted extinction in terms of six distinct network attributes 14 resulting from generalists-first (red) and specialists-first (green) extinction sequences 15 are plotted against the fraction of nodes removed. These six attributes are (a) Species 16 richness (b) Secondary Extinctions (c) Nestedness (d) Fragmentation (e) LDia and (f) 17 NDia. Note the coupled variations between panels d-e-f at a given point on the $\mathrm{X}$-axis. 18 In the generalists-first scenario, when NDia is at its lowest, the reduced network 19 fragments into disconnected subwebs (by 10\% loss) followed by rapid collapse. 20 However, in case of specialists-first, whenever the NDia reaches a minima, there 21 occurs a compensatory reduction or 'flip' in LDia, causing an immediate recovery in 22 NDia values, and the unfragmented nature of the network is preserved.

\section{Figure 3}


1 Variation between NDia and LDia in eight representative ecological networks

2 following extinction cascades, resulting in flips and alternate stable states. Panels $a$

3 and $b$ depict two seed dispersal networks (SILV, JOR1) while panels $c$ to $h$ depict one

4 each of pollinator (MEMO), Anemone-Fish (ANEM), Ant-Plant (BLUT), Host-

5 parasite (LAKE), Plant-herbivore (JEOM) and Predator-prey (MART) network. For

6 each network, the panels contain the corresponding NDia and LDia plots arranged

7 vertically below each other. As in Figure 2, each plot depicts the change in the

8 respective network attribute as a function of the fraction of nodes removed. Note that

9 each drop in LDia corresponds to a surge in the NDia for a given web. The

10 length/duration of each stable state can be measured as the fraction of nodes removed

11 while the LDia remains constant. The number of states can be measured as the

12 number of stable flips in LDia. Details for each network are provided in

13 Supplementary Data A2.

14 Figure 4

15 Influence of initial LDia of a network on its 'resistance to flip' or the primary 16 extinctions required for a flip. The box plot provides a summary of the observations 17 for 86 networks analysed in this study (a). Networks with smaller initial LDia show 18 resistance to flip compared to networks with larger LDia as more primary extinctions 19 are needed for a state change. Loess of the same (b) suggests a curvilinear rather than 20 linear nature of the relationship. The larger networks have lower resistance to flip 21 indicating that fewer primary extinctions can cause a state change in their 22 communication levels.

23 Figure 5 
1 A schematic framework explaining the alternate states following a specialist first

2 extinction cascade in ecological networks. This is a schematic representation using

3 GNIC as the baseline. The X-axis corresponds to percentage primary extinctions with

4 specialists being removed first along a specialist-generalist continuum. The Y-axis

5 corresponds to the number of diameters or NDia values of the network. Each state is

6 depicted as a grey arc. For instance, $\mathrm{x} 1$ corresponds to the unperturbed status of the

7 network (percent primary extinctions $=0$ ) and $\mathrm{x} 2$ corresponds to the threshold value of

8 percent primary extinction beyond which a flip (shown by bold arrows) occurs to the

9 next state. The range of NDia values that correspond to the primary extinctions (y1 to

$10 \mathrm{y} 2$ ) characterize the resilience of the network as it maintains its state (LDia=6) until it

11 reaches a threshold of extinctions at $\mathrm{x} 2$, beyond which a state change to lower

12 diameter $(\mathrm{LDia}=5)$ occurs. This shift is path dependent since addition of a node at this

13 stage is unlikely to bring about a reversal of the state (see discussion), resulting in

14 hysteresis as state changes occur repeatedly in the cascade. 
Figure 1

2

(a)
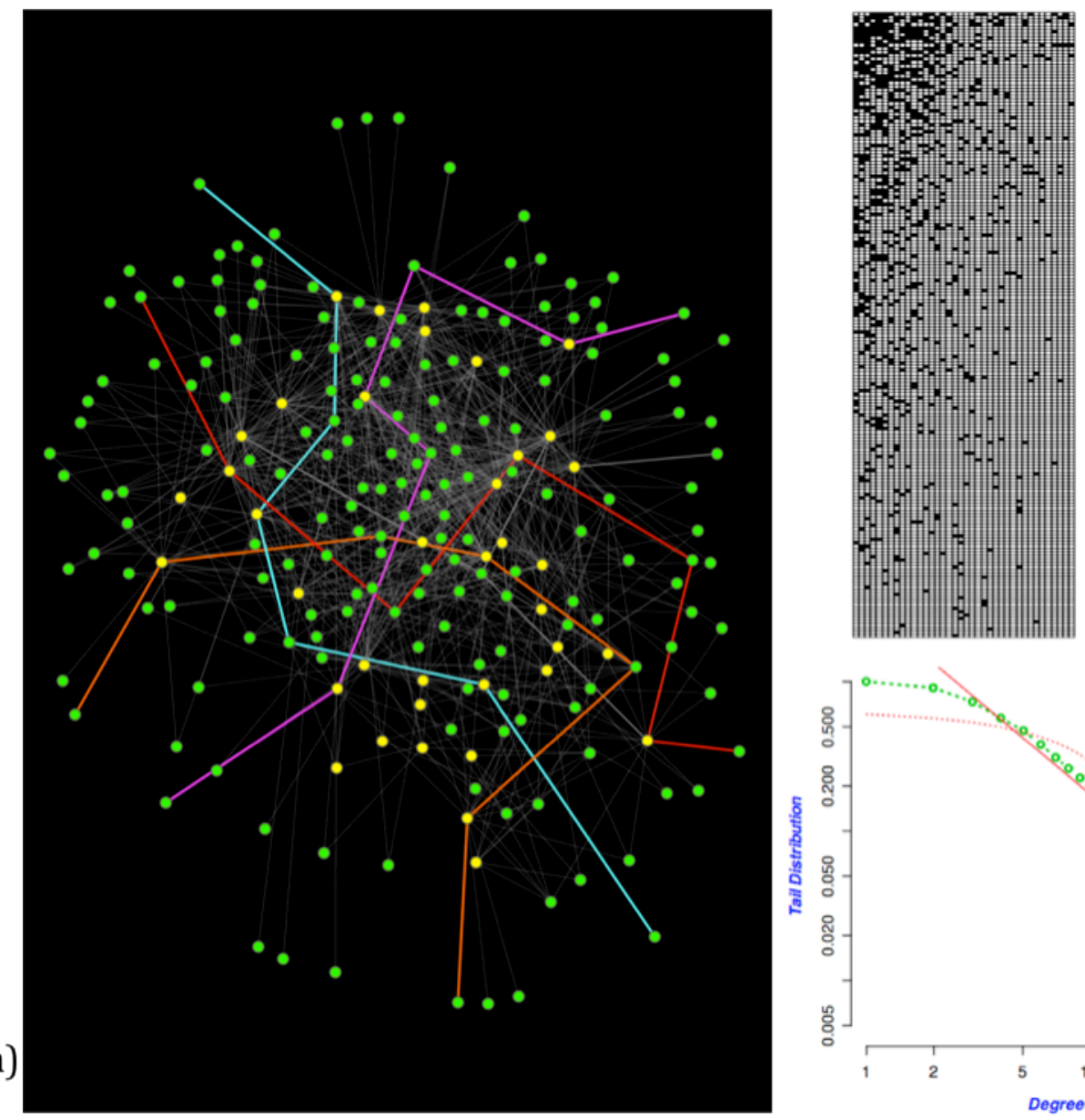

(b)

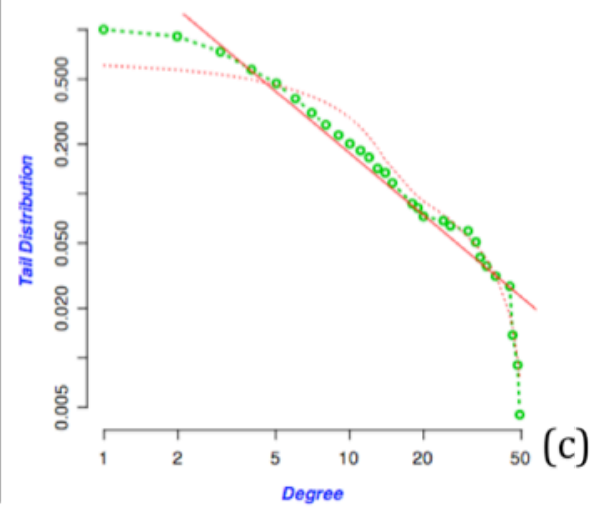



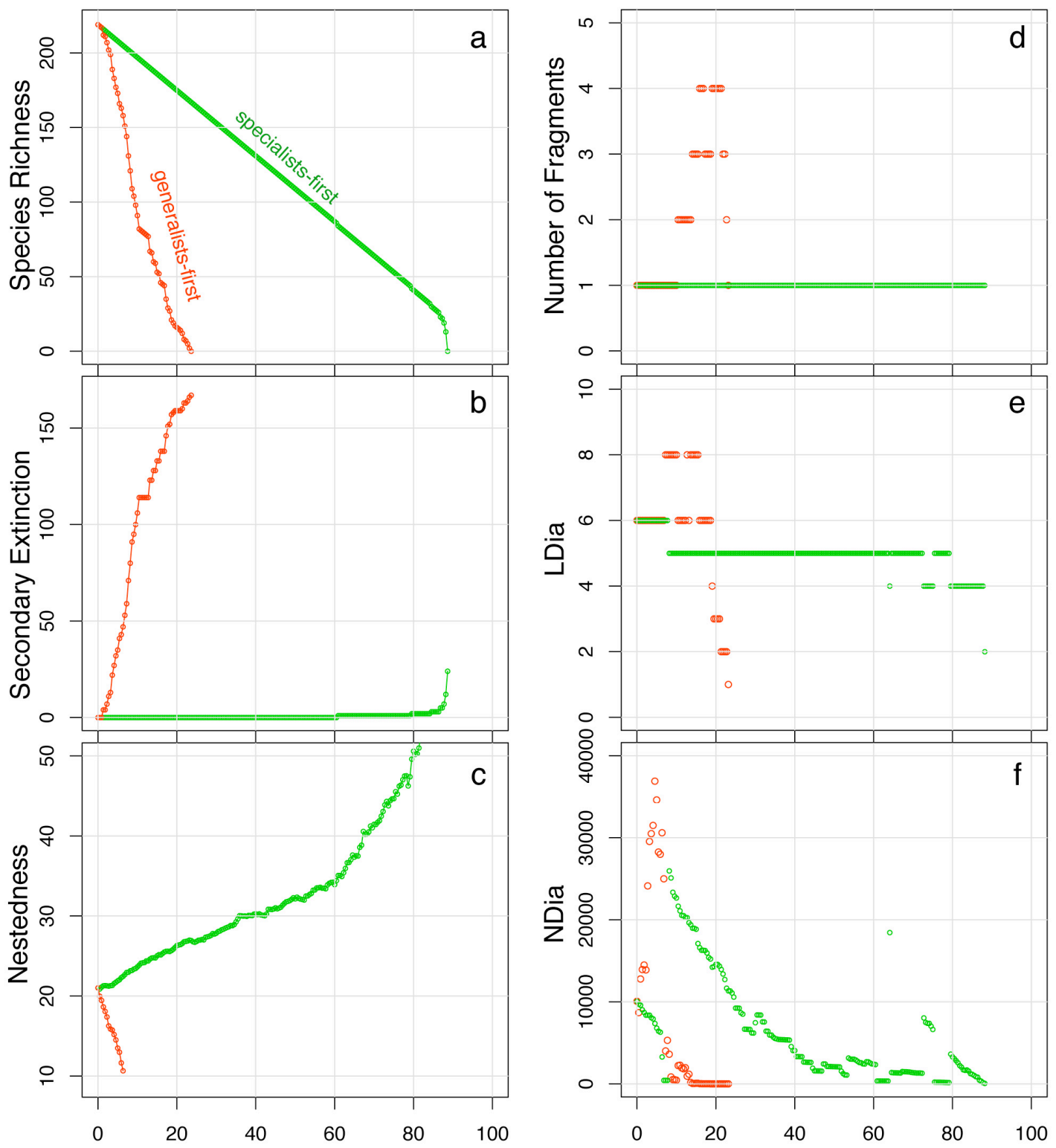

Percentage Removed Nodes

Figure 3 

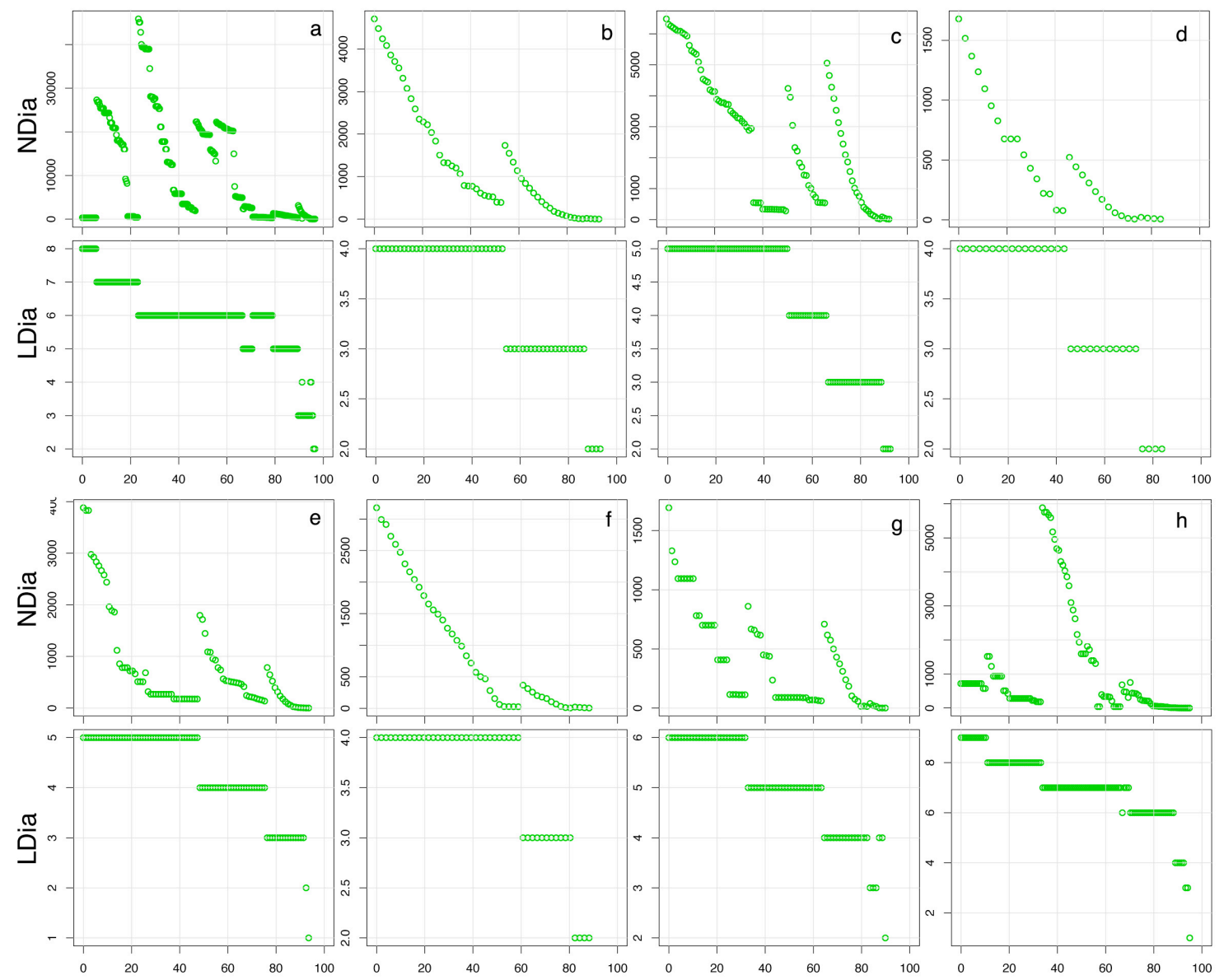

Percentage Removed Nodes (Minimum to Maximum based on degree) 


\section{$1 \quad$ Figure 4}

2
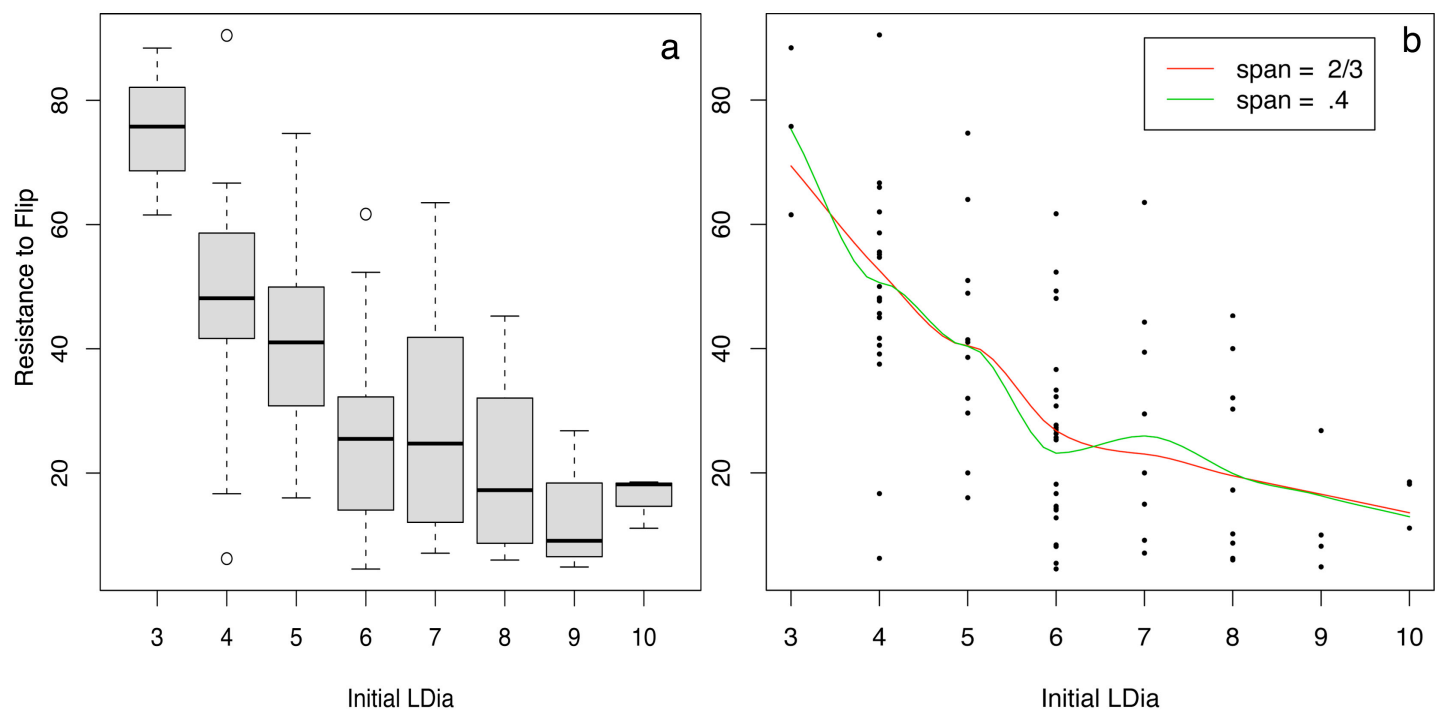

3

4

5

6

7

8

9

10

11

12 


\section{Figure 5}

2

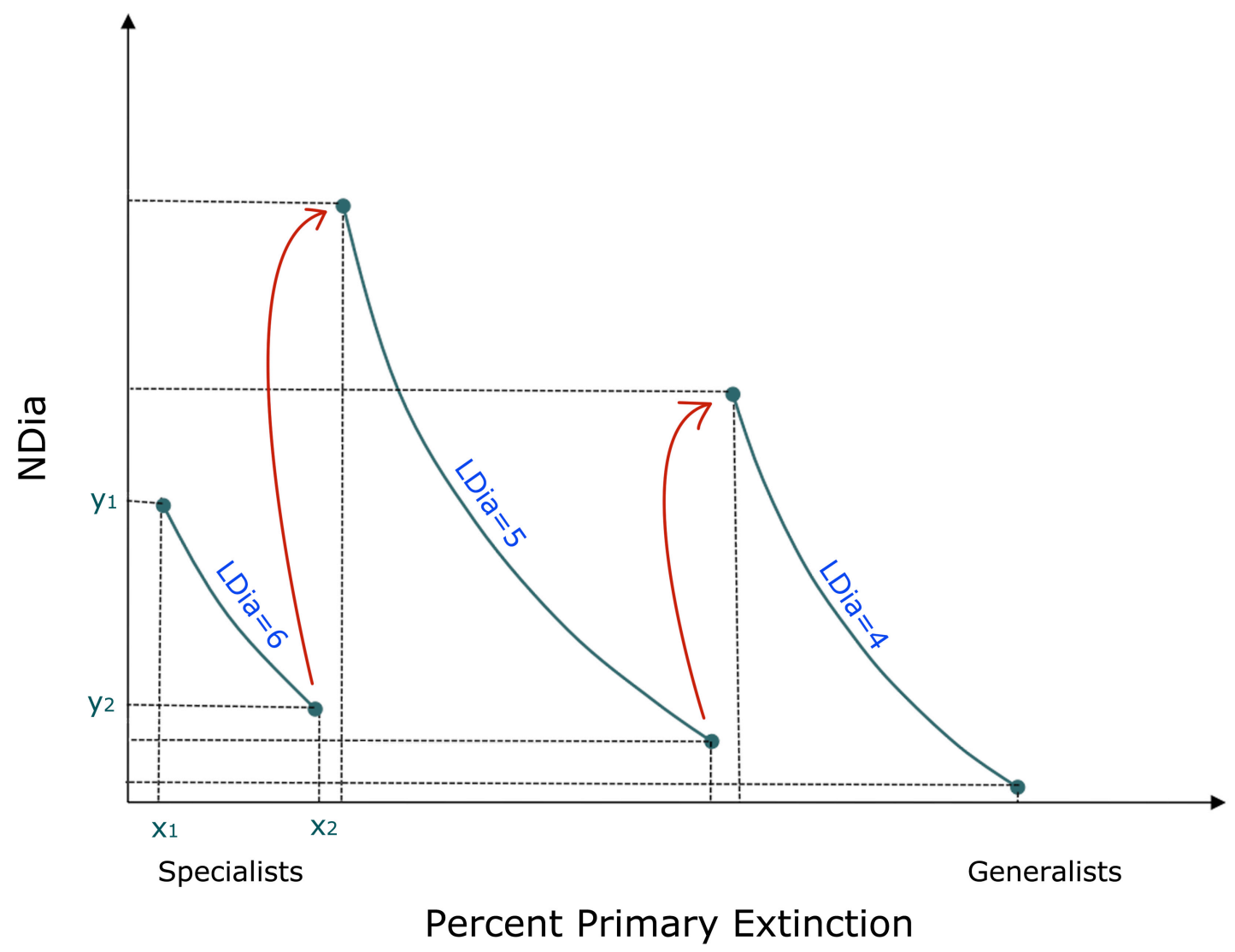

3

4 